\title{
A physically-based and distributed approach to analyze rainfall-triggered landslides at a watershed scale.
}

\author{
E. Arnone (1), L.V. Noto (1), and R.L. Bras (2) \\ (1) Dipartimento di Ingegneria Idraulica ed Applicazioni Ambientali, Università di Palermo, Palermo, Italy \\ (elisa.arnone@gmail.com, valerio@idra.unipa.it/+39 0916657749), (2) The Henry Samueli School of Engineering, University \\ of California, Irvine, USA (deansofc@ soemail.eng.uci.edu )
}

Landsliding phenomena represents one of the natural hazard causing serious threat to both lives and property in all the world. Failure is due to multiple dynamic processes, including rainfall and hydrological processes, so that knowledge of spatio-temporal hydrological processes is the first step for the analysis of landslides triggered by rainfall. Mapping areas susceptible to landslides should be the result of this kind of analysis to support land-use management decision-making.

In scientific literature, many methods and techniques have been proposed to estimate when and where failure could occur. Integration of a failure model with an hydrological model allows simulation of rainfall-triggered landslides, taking into account spatial and temporal hydrological dynamics influencing the landsliding, first of all the soil infiltration, evapotranspiration, groundwater dynamics, wetting soil conditions.

The aim of this paper is to describe a landslide component of a physically based and spatially distributed hydrological model - tRIBS. This component attempts to model rainfall-triggered landslides by implementing the infinity slope model into the hydrological model tRIBS that provides the necessary data to define the pore pressure conditions. Furthermore, the component inherits by the tRIBS the multiple resolution approach (TIN geometry) to represent the spatial heterogeneity of topography that offers a reduction of the computational elements without any loss of information. For each irregular cell, the model is able to provide the stability condition as a function of the Safety Factor (FS), as well as to estimate the amount of detached soil and its path.

First test of model performance is evaluated carrying out a setup case in a small catchment with very steep slopes, located in the northern part of Sicily. The component is still at a developmental stage and this first application is useful to emphasize weakness and strength of the model as well as to enhance the component. 